\title{
The Research on Basic Parameters of Ball Spinning Technology Characteristics
}

\author{
Zhanjun Liu \\ Faculty of Aerospace Engineering,Shenyang Aerospace University,Shenyang ,China \\ luzluzd@126.com
}

Keywords: ball spinning; ratio; thinning; basic parameters; efficiency

\begin{abstract}
The unique characteristics of thin ball spinning technology is better than wheel spin, tube can choose a larger one including a reduction ratio, it usually includes a reduction ratio that can reach $40 \% \sim 70 \%$. Therefore, it can greatly improve the production efficiency. However, including a reduction ratio, the material can spin and spinning equipment capability on craft, except that the restrictions are spinning pipe quality and accuracy requirements. Metal material is expected maximum spin, the limit of thinning rate depends largely on inherent plastic metal materials.
\end{abstract}

\section{Introduction}

Ball spinning is a branch of powerful variable pressure, in addition to the other spinning method characteristics, but also it has the unique feature of the process, and it is the process in the past 30 years, which is in the field of civil and military industry and so on, production and processing precision is small diameter of thin-walled tube obtained increasingly wide application. Ball spinning technical and economic characteristics are as follows:

It is good fitting surface roughness and high dimensional accuracy. Because the transform tool ball has good surface roughness and high dimensional precision, the deformation mode is point by point, and elastic deformation is small, to ensure the spiral pipe diameter size that can be controlled within $0.005 \mathrm{~mm}$. The surface roughness is usually up to $0.2 \mathrm{um}$. Process configuration and the product of the surface roughness can be almost near with after grinding the surface of the product. The wall is up to the thinnest of pipe fittings. Ball spinning of these traits are usually rolled, rollers are hard to reach.

\section{The Ball Spinning Process Technology Characteristics}

The metal pipe mechanical properties was improved. Metal pipe fits in the deformation zone in compressive stress state, deformation is after the prolongs material grain and groups of woven refinement, which is with continuous fiber structure, it improve the yield strength of metal, tube hardness is as well improved, elongation is reduced[1].

The process method is simple, easy to grasp. Spinning process does not require relying too much on the operator of intervention technology, technology and equipment is simple. Usually cutting machine is to improve slightly, which can be used in ball spinning processing. Due to the deforming force is small, small tonnage equipment is used to processing large size products.

The mold consumption is low. Ball spinning is compared with deep drawing drawing, because the spinning deforming force is small, and the rolling friction insteads sliding friction, the ball spinning mould of more general can adjust the size range, the mold consumption is only 1/5-1/8 for stamping drawing die[2].

The deformation is high efficiency. For the plastic and alloy of general good gold scrap, the reduction of area of the single channel time can reach $70 \% \sim 85 \%$. While drawing pipe is only about $30 \%$. If compared with the cutting, it can improve production efficiency of 30 to 50 times. It makes it easier for small batch more varieties, specifications length and diameter of the large one. Therefore, ball spinning is deformation of thin, which force is not only small, and spin after fitting surface is of high quality[3]. Due to the required load of ball spinning is small, it can take advantage of small tonnage equipment to process large size pipe fittings. 
Moreover, because the quantity of application more ball is full of concave die. The process of deformation and stress tends to be more uniform, to prevent the instability of the process of spinning and distortion. The radial component of tension and compression is more balanced, to reduces the pipe hole enlargement. It ensures that the ball spinning tube has a higher accuracy than the wheel pressure.

Ball spinning of processing and application range is 3 to $150 \mathrm{~mm}$ in diameter, the tube wall thickness is less than 3 to $5 \mathrm{~mm}$, the finished wall thickness is $0.1 \sim 1.2 \mathrm{~mm}$, usually up to $0.04 \mathrm{~mm}$ of the thinnest. Due to production product has the high accuracy and good mechanical properties, and the advantages of saving time has saving raw material, thus ball spinning pressure in the aviation, aerospace, electronics and instrumentation, and which won the growing medical apparatus and instruments[4].

\section{The Basic Parameters Of Spinning Technology Is Set}

3.1 The wall thickness of reduction ratio. Reduction ratio is the important process parameters in the process of ball change, because it directly affect the spinning force size, production efficiency is high, and the stand or fall of fitting is accuracy[5]. Thinning is always lose.Thinning ratio passes two slaughter. The total reduction ratio is of logarithmic reduction ratio for different combined. In the process of ball spinning thin, it is the time to become pressure reduction ratio of influence and choice.

Because the unique characteristics of thin ball spinning technology is better than wheel spin, tube can choose a larger one including a reduction ratio, it usually including a reduction ratio that can reach $40 \% \sim 70 \%$. Therefore, it can greatly improve the production efficiency. However, including a reduction ratio, the material can spin and spinning equipment capability on craft, except that the restrictions are spinning pipe quality and accuracy requirements[6]. Metal material is expected maximum spin, the limit of thinning rate depends largely on inherent plastic metal materials. Ball spinning thin thinning rate is expression. This is because the pressure has the following features:

It improves the spinning metal liquid, if spinning process parameters selection is undeserved, it can cause deformation zone before the metal accumulation, wall instability produces the disadvantages such as hole enlargement. To improve the process parameters, it usually lower the spinning production efficiency. And after the pressure, it can make gold flow conditions greatly improved. This can increase the wall thickness of reduction ratio noise and reduce skin pile product, to improve product quality and production efficiency.

The pipe shape and dimension precision was improved after spinning. The existence of axial tensile stress can be suppressed to tensile stress, it is not easy to produce hole enlargement. Tensile stress is easy ball bearing deformation area before fitting the mobile, the prompting metal accumulation is reduced, supination are more likely to die pipe fittings. It makes rotary pressure decreased significantly. Fig. 1 is shown as curve of carbon steel pipe without tension axial force. Fig. 2 is curve of carbon steel pipe of toughness and hardness .

The figure is shown that the use of tension has significantly reduced than without tension. And the decrease depends on the axial feed rate, wall thickness thinning, tension values, and materials performance, etc.In a word, experimental study and production practice shows that application with pressure method is to produce one specifications, an effective method is high precision, and high surface quality is thin-walled tubes.

3.2 The ball spinning of ultrasonic vibration. Generally the vibration effect is considered to be harmful, such as the lower is for accuracy, it not impacted equipment function, which shorten the service life of the mechanical, etc. However, the vibration also achieved a lot special applications, such as vibration polishing, vibration grinding, welding, vibration aging, etc. The austrians found that using the ultrasonic vibration can be observed, when stretching the material, yield stress and flow stress reduce the phenomenon. Up to now, after decades of development, the ultrasonic wave obtained wide application in the plastic forming process. As in the wire drawing, extrusion and blunt, the application of pressure is more mature, also spinning processing is application promotion. 
The ultrasonic spinning, that is the ultrasonic frequency electromagnetic oscillation wave came into a corresponding frequency rate of mechanical vibration wave, which spread to spinning and tools, applied to deformation zone.

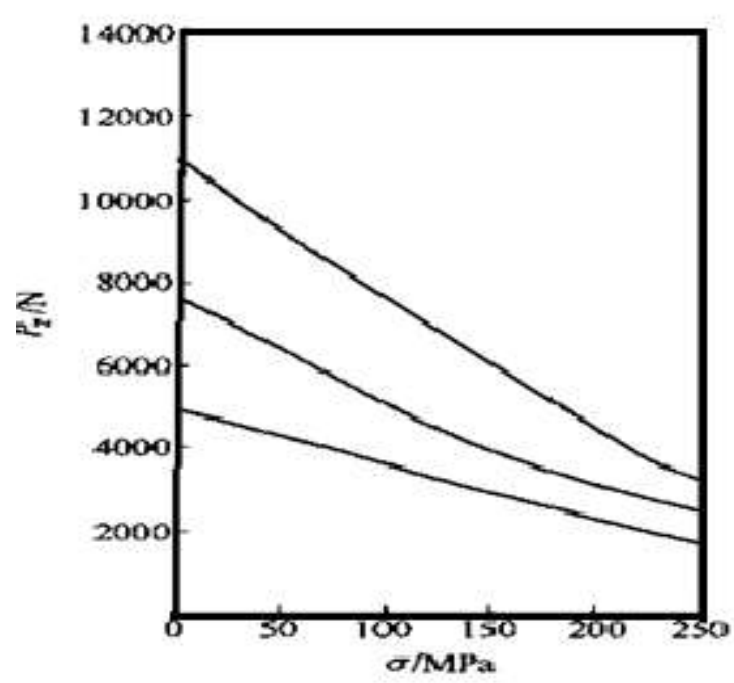

Figure 1. Curve of pipe without tension axial force

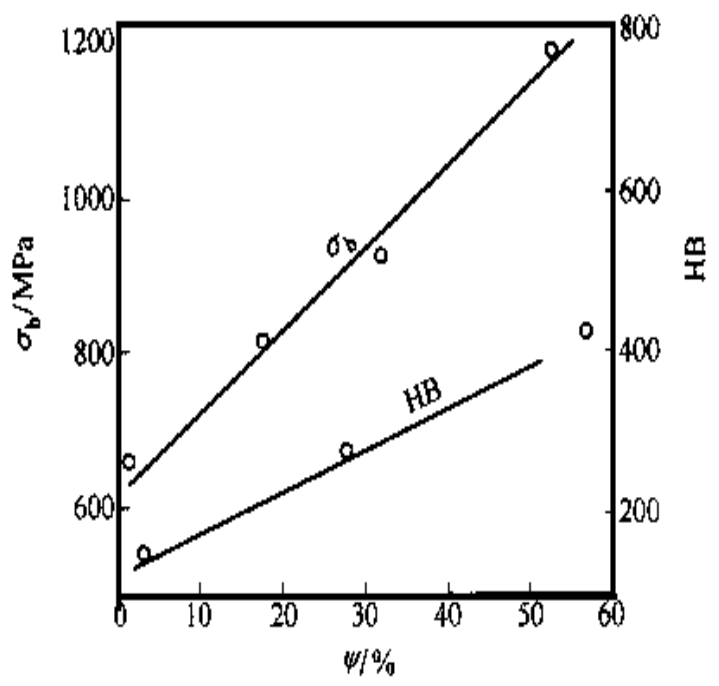

Figure 2 .Curve of toughness and hardness

In order to produce some beneficial to metal plastic deformation, effect is the technological process. About ultrasonic vibration is move on the surface of the plastic materials and the influence of internal theory, all is based on the body product effect and surface effect two concepts. On the basis of internal particles, blank is produced after vibration material activity increases, temperature rise is in connection with the crystal dislocation thermal softening.

The dynamic deformation resistance reduced. The second is the ultrasonic vibration on core mold, the die and billet separation between vibration is producing instant reduce of external friction, vibration is also improved the spinning processing of slippery conditions. The application of ultrasonic vibration, in short, reduces the forming force when plastic processing reduce the mold and artifacts friction and improve the product surface quality, etc.

In thin small diameter spiral pipe, pipe inner surface usually has a very small gap between mandrel, the tangent deformation can be ignored, which can be treated as plane strain state. Pulling a pipe from the deformation zone is this section of the tube, and billet is in contact with the mandrel surface, and again spinning ball spherical contact. Strictly speaking, the axial cross section of deformation zone is a circle radius, therefore, the deformation zone can be viewed as cone angle, it is equal to the axial spinning of meshing angle conical surface.

Because the ball spinning is defined as a plane strain state, the mean principal stress is equal to the critical half of the principal stress. Effect of pressure on the pipe wall thickness direction. Stress is normal maximum principal stress. From spinning tube billet is deformation zone. Distribution of force values of principal stress in the deformation zone is a variable. Axial force should be little value in deformation, which is a number of different factors. Not only from the qualitative aspect, but from the quantitative aspects. Study is all the factors that affect the deformation resistance, so as to seek the precise rules and deformation resistance.

Accuracy production efficiency has a close relationship. Therefore, grasps is the spinning force calculation and determination methods, such as, to increase productivity, reduce energy consumption, and promote science and technology progress, and to improve the tooling and equipment is very important.

Metal and alloy plastic objects exist a kind of state. It not only depends on the object the nature of the internal and external conditions, also depends on the deformation. Such as stress deformation mode decided 
state, degree of deformation and temperature of the metal processed, etc., all have not suddenly on metal plastic visual impact.

\section{Conclusions}

The unique characteristics of thin ball spinning technology is better than wheel spin, tube can choose a larger one including a reduction ratio, it usually includes a reduction ratio that can reach $40 \% \sim 70 \%$. Therefore, it can greatly improve the production efficiency. However, including a reduction ratio, the material can spin and spinning equipment capability on craft, except that the restrictions are spinning pipe quality and accuracy requirements. Metal material is expected maximum spin, the limit of thinning rate depends largely on inherent plastic metal materials.

\section{References}

[1] M. Abdalla, J.H. An, M. Bellare and C. Namprempre. 2008. From identification to signatures via the Fiat-Shamir transform: Necessary and sufficient conditions for security and forward security, IEEE Transactios on Information Theory, 54(8): 3631-3646.

[2] J.H. An, Y. Dodis and T. Rabin. 2002. On the security of joint signature and encryption, In Advances in Cryptology-Eurocrypt 2002, 2332: 83-107.

[3] B. Barak and M. Mahmoody-Ghidary. 2007. Lower bounds of signatures from symmetric primitives, In 48th Annal Symposium on Foundations of Computer Science, 290(1-2): 680-688.

[4] M. Bellare and S. Shoup. 2008. Two-tier signatures from the Fiat-Shamir transform with applications to strongly unforgeable and one-time signatures, IET Proc.Information Security, 2(2): 47-63.

[5] D. Boneh. 2008. Short signatures without random oracles and the SDH assumption in bilinear groups, Journal of cryptology, 21(2): 149-177. 